\title{
What Can You Do To Virtually Teach Hands-on Skills?
}

\author{
Peter Fenrich \\ British Columbia Institute of Technology, Burnaby, Canada \\ peter fenrich@bcit.ca
}

\begin{abstract}
What can you do to virtually teach the hands-on skills traditionally taught in labs? If you include simulations, active experimentation, discovery-learning techniques, numerous questions with detailed feedback, video, animations, and photographs, you can effectively teach practical hands-on skills through multimedia technology. Through discussion and demonstration, this session will highlight practical tips for implementing the instructional development cycle as well as uncommon but effective instructional design strategies for teaching practical skills. Some of the highlighted programs (such as a virtual chemistry lab) have pushed the boundaries of what can be accomplished with multimedia technology. By the end of this interactive session, participants (who can range from novices to experts) should be able to identify computer-based training applications that effectively use multimedia technology, generate examples of where new media technology can be appropriately used to virtually teach hands-on skills, and decide where to incorporate the strategies shown into their computer-based training productions.
\end{abstract}

Keywords: virtual lab, practical skills, instructional design, computer-based training, interactivity

\section{Introduction}

Many of the challenging computer-based training/simulation projects undertaken by the Group for Advanced Information Technology (GAIT) at the Technology Centre of the British Columbia Institute of Technology have taught practical skills. These projects have been initiated because clients or instructors have identified those areas as being problematic. In other words, there are some significant limitations when the skills are attempted to be taught via traditional methods:

1. Learners may not be able to attend teaching/training locations.

This is an inherent problem in distance education. Many learners can learn the theory online or through other distance education solutions. However, they may not be able to learn the needed practical skills at a distance. Virtual labs can solve this problem. A demonstration version of an award-winning product that teaches both the theoretical and lab components of the reaction kinetics portion of high school chemistry can be downloaded from

Material published as part of this journal, either on-line or in print, is copyrighted by Informing Science. Permission to make digital or paper copy of part or all of these works for personal or classroom use is granted without fee provided that the copies are not made or distributed for profit or commercial advantage AND that copies 1) bear this notice in full and 2) give the full citation on the first page. It is permissible to abstract these works so long as credit is given. To copy in all other cases or to republish or to post on a server or to redistribute to lists requires specific permission from the publisher at Publisher@InformingScience.org

www.bcit.ca/appliedresearch/gait/proj ects/reactionkinetics.shtml Given the need for distance students to learn practical skills, a current GAIT project underway is the creation of a virtual biology lab that teaches all of the lab skills taught in first-year biology. It is 
scheduled to be completed by March 31, 2006.

2. There may be an inability to teach some content well.

This can be apparent in topics where effective learning requires simulations. For example, one project was teaching piping students how to test and troubleshoot backflow prevention assemblies. A demonstration version of this project can be downloaded from www.construction.bcit.ca/trades/piping/computertraining.shtml

3. Logistical challenges can be problematic.

Logistical challenges can arise when experts do not have enough time to travel around the country to teach (i.e., their fulltime job does not leave them with enough time to provide training). One project entailed capturing the expertise of personnel in a spinal cord injury centre of a major hospital on how to move and turn patients with a spinal injury. The resulting program was then freely distributed on CD-ROM and web download to those who needed the training around the world. This award-winning project can be freely downloaded from www.bcit.ca/appliedresearch/gait/projects/spinal.shtml

4. Costs for live training can be prohibitive.

Live training can be very expensive. For example, costs are high when numerous participants are required to travel to a workshop. One project involved training aircraft mechanics. Costs typically included salary for one day to travel to the workshop location, two days at the workshop, and one day to return home as well as travel, accommodation, and per diem expenses. The resulting CD-ROM allowed mechanics to learn when and where they wanted in approximately one-quarter of the time needed in the workshop.

5. Equipment can be a limitation.

The equipment itself can be a limited resource. For example, one way to teach troubleshooting involves taking components out of functioning systems, breaking them, putting them back in, and letting students determine the problem. However, this is impractical because of the time it takes to remove, break, and install components, the cost of later repairing the components, the problem that this needs to be done to many components, and the time required to have all of the students individually determine the problem for each broken component. One simulation was created to address this type of equipment limitation. It entailed teaching piping students how to troubleshoot hot water heating systems. A demonstration version of this project can be downloaded from www.construction.bcit.ca/trades/piping/computertraining.shtml

6. There may be a requirement for just-in-time learning.

For example, can be needed when instructors are hired in post-secondary settings. It is not unusual for the individual to have had no training in how to teach as they are mainly hired for their subject-matter expertise. However, they often have to begin teaching before a workshop is available or any formal training can be delivered. GAIT has created a highly-interactive product to deliver basic instructional skills where and when an individual needs it.

The instructional challenge in these projects has been to ensure that the practical skills taught via the computer transfer to the real world. There is evidence to support that this can be done (Fenrich, 2002).

Note that, in this context, virtual labs simulate and teach what learners must do in traditional settings. A virtual lab is not created simply because one has transferred their lab workbook to a website. 


\section{Problems with Teaching Practical Skills in Traditional Classrooms}

There can be numerous problems when teaching practical skills in typical classrooms and labs:

- When a demonstration is done, some students may not be able to clearly see what is being demonstrated (e.g., those in the back of the class).

- Many instructors will not show all of the possible demonstrations (due to time or cost considerations).

- In many cases, if a student misses an activity, they will not be able to do it later.

- Students are often not able to repeat an activity if something goes wrong.

- Dangerous, expensive, or unavailable equipment or materials may limit what learners can see or do.

- Quite often, media is needed to teach practical skills. Existing media may not be easily shown when and where a student needs it. For example, a repairperson may need to see video clips while they are working on the equipment rather than in the classroom itself.

- Some existing traditional teaching materials are not effective. For example, a 20 minute videotape was created to train student mechanics how to disassemble and assemble an aircraft engine. However, the total disassembly and assembly requires approximately 200 steps. A better instructional solution would have included step-by-step short video clips and text explanations that can be accessed while the student was practicing on the engine.

- Even if a student is fortunate enough to be taught with media (when it is instructionally required), questions and feedback are often delivered via text. In many cases, if the content needs to be taught with a variety of media, effective testing requires a variety of media.

Given solid instructional design strategies, multimedia technology can and has solved these limitations.

\section{Instructional Development Cycle}

In general, the design and development of software that teaches practical skills (as with other types of instructional software) should follow an iterative instructional development cycle as illustrated in Figure 1. The presented instructional development cycle is adapted from Spitzer (1991). Following the instructional development cycle is essential in helping create effective instructional multimedia solutions. Many projects have failed because steps in each phase have not been followed or were not done well enough. 


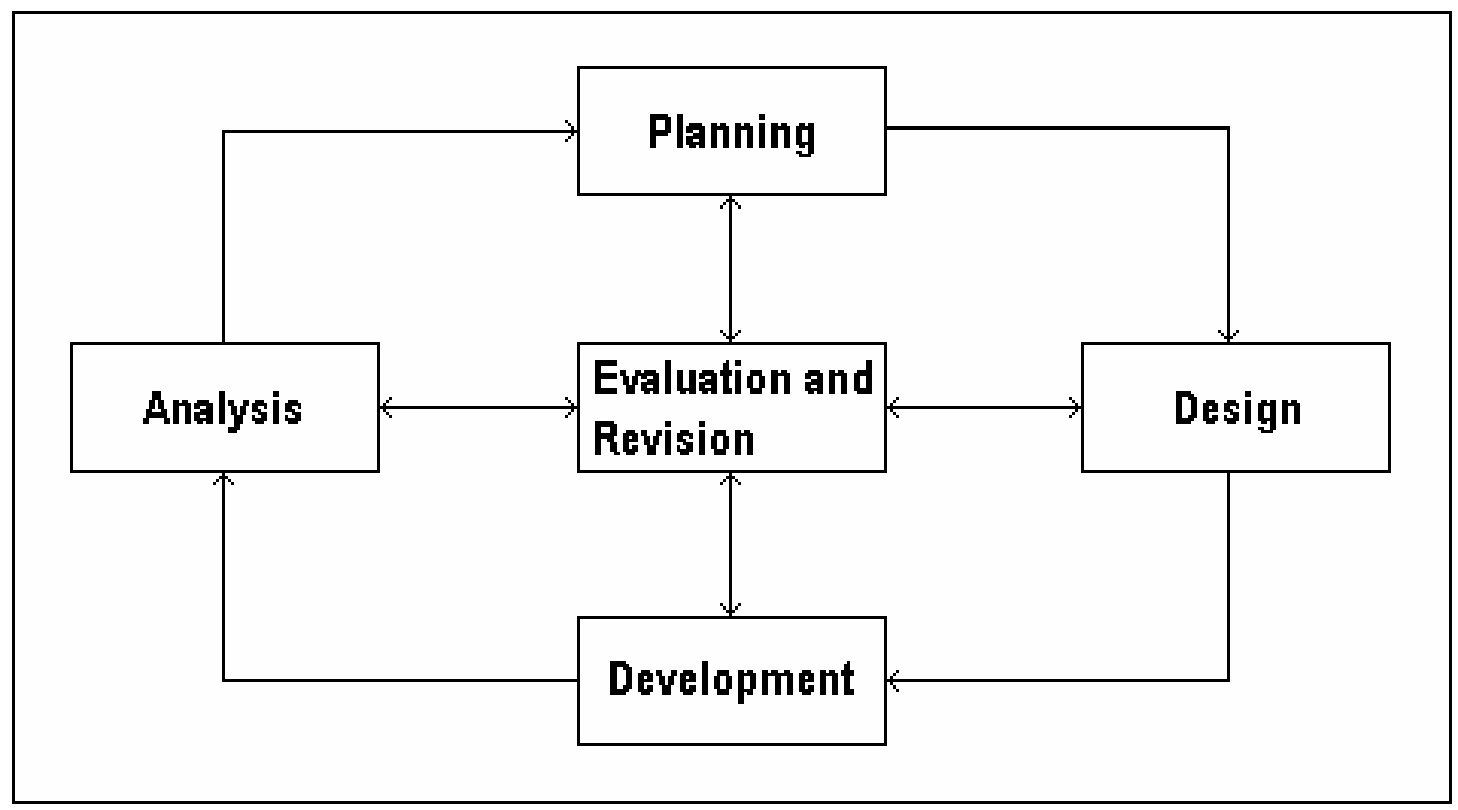

Figure 1: Model of the Instructional Development Cycle (Adapted from Spitzer, 1991)

\section{Analysis Phase}

The initial purpose of the analysis phase is for you to define the actual problem and then determine whether the problem needs an instructional multimedia solution. The analysis phase should provide you with a concise problem definition and initial cost estimates (Fenrich, 2005).

The time invested in the analysis phase tends to save more time throughout the rest of the process. If you do not spend enough time properly defining your learning outcomes, the entire subsequent development could be for unneeded skills. For example, be sure to identify the actual problem rather than symptoms of a problem. The symptom may be a refusal to use a software program because the "program does not work" but the real problem is a fear of the technology. So, an instructional solution aimed at how to use the software could be a wasted effort.

Well-written learning outcomes help to keep the subsequent instructional development process on track. When in doubt, always refer back to the stated learning outcomes. It is often tempting to cover interesting but unneeded or non-funded topics. For learning outcomes, also consider what the learner really needs to learn rather than what one wants to teach.

More specifically, for practical skills, determine what the learner actually needs to do. One way to do this is by imagining what skills the learner needs in the real world. For example, in some cases in the laboratory portion of chemistry, the learner does not really need to pour one chemical into another. The important skills may be the observations, data analysis, and conclusions that are drawn. For virtually teaching practical troubleshooting skills, your analysis may indicate that the critical skill that a learner may need to learn is to analyze the interrelationships between the components of the system. Regardless, of the application, your instructional multimedia solution should simulate the actual practical skills needed.

It may be wise for you to inflate your initial cost estimates in order to be sure you can complete the project within your budget. It is safer to assume that teaching practical skills requires a higher level of instructional design (as compared to developing traditional materials) to ensure learning, the computer programmer will have to develop complex programming code that does not fit within existing templates or subroutines, and more media than is usually developed. 


\section{Planning Phase}

The planning phase helps your whole project proceed smoothly. A major component of the planning phase involves identifying and addressing potential problems. Other major planning tasks include assembling the team and setting timelines (Fenrich, 2005).

Problems could be a lack of acceptance from all levels (e.g., for creating a virtual lab, some individuals may feel threatened by its creation), inexperienced team members, lack of development tools ... If you are attempting to create a complex simulation, such as a virtual lab, it is even more important to have all potential problems solved before investment in design and development have begun. In particular, be sure that you have skilled resources and funding in place.

\section{Design Phase}

Based on your problem definition and the stated learning outcomes, the design phase leads you to creating the instructional strategy - what needs to be done to ensure that the students will effectively and efficiently learn. Another component of the design phase is to define the standards and needed utilities so that programming can begin. In this phase, create programming sub-routines and templates. These can save an enormous amount of time during the development phase. A last step is to finalize all of the resource requirements (Fenrich, 2005).

As much as possible, to save time and money, base new projects on templates and subroutines that have been created for other computer-based training applications. However, do NOT compromise learning simply for the convenience of using templates. You should expect that only a limited amount of a virtual lab or simulation will follow existing templates.

Design an intuitive user-interface based on the philosophy: If you have to explain how to do it, you have done something wrong. An intuitive user-interface is particularly important in virtual labs and other simulations. Too many existing simulations have had minimal results or have failed due to their complex user-interfaces.

Determine how to guarantee effective learning! This is a particular challenge when students are on their own and cannot have their questions immediately answered. Think outside the box to guarantee learning. Consider simulation, discovery-learning techniques, and active experimentation. Page-turning activities will not suffice for learning some practical skills. Use the technology for its strengths rather than simply transferring content from one format to another! A problem with this task is that there is no single formula to follow. Designing the instructional strategy is both a science, such as following Dick and Carey's (1990) principles of instructional design, and an art. Different learning outcomes may need different instructional strategies. No single strategy will successfully teach all learning outcomes. Again, think outside the box. Consider including simple games to teach practical skills as games provide a fun way to learn (McFarlane, Sparrowhawk, \& Heald, 2002).

Think about the limitations of teaching each skill virtually. For limitations, you will have to realistically determine what level of skill you can achieve. For example, in a virtual biology lab, how will you teach a student to learn how to use a microscope? This is a limitation of using virtual technology. However, a lot can be done with video. For example, you could show the course adjustment being adjusted, then what the learner would see inside the ocular lens, then show the fine adjustment being adjusted, and then what the learner would see inside the ocular lens. A practical activity could have the learner set the course and fine adjustment to find the clearest image. The learner could click on arrows to move the course and fine adjustments up and down and see the corresponding image of what would be seen. Similar video clips and activities can be used for demonstrating other microscope components such as the condenser lens. This would not be as good as what is done in a real lab but would definitely give a sense of how a real microscope 
works. Other activities could potentially lead to better results than a real lab. For example, in a real lab, students see specimens and are then asked to draw what they see so that they can later study from their lab book. In a virtual lab, students will see full-color video clips and photographs but they can also later study them when they prepare for their lab test. These virtual images would be better review materials than what the typical student would see from their drawings made in a live lab. One key in creating a successful virtual lab is getting as close as possible to reality (given constraints of time and money) for skills that will be weaker than live labs but balancing these with activities that can be better than live labs.

Organize the information into small enough chunks for the students to successfully learn. For example, if the procedural skill has numerous steps, consider teaching them in logical groups of between three to six steps (Dick \& Carey, 1990). Many instructional resources, which attempt to teach practical skills, provide too much information or too many steps for students to learn at one time.

Base some of the content on the potential for making mistakes (Fenrich, 2005). Ask the content expert about typical mistakes made after the content is taught in the traditional way. If one only teaches what is correct, the learner may never learn what can go wrong. For example, teaching what can go wrong is important in teaching physicians how to make an accurate diagnosis.

To address a variety of learning styles, teach with a variety of different methods and media. No one single teaching method or medium is perfect for all learners (McCarthy, 1987). Note also that teaching with more than one medium is usually more effective than teaching with only one medium (Romiszowski, 1988). Teaching practical skills via computer usually requires a variety of media. Use media to enhance learning. Do NOT include media (e.g., animations) simply because it can be done. It can be a significant waste of resources. This is a particular concern when resources are limited.

Note that practice questions can include media that are similar or identical to those used in the presentation. Assume that if the content needs to be taught with media, the content should be tested with media (Fenrich, 2005). For practical skills, it is likely more accurate to test with a photograph or video clip than text alone. Remember that a picture is worth a thousand words. This is particularly true for hands-on skills.

Determine whether testing is realistic enough and a true performance measure. This is particularly important for practical skills. When testing, consider all difficulty levels (Mayer, 1988). Many existing computer-based training packages are weak in that they only address low-level thinking skills rather than the actual skills needed.

Make the program highly interactive throughout! Interactivity is active participation in the learning. Interactivity requires the learner to think (Fenrich, 2005). It is easy to create interactions when you virtually teach practical skills. You can do this in a number of ways including having students drag and drop items, increase and decrease settings to observe results, make decisions, answer questions based on video clips and photographs that show correct and incorrect procedures or results... Remember to always provide detailed feedback, even when the answer is right. This is in case the student guessed the correct answer or answered correctly for the wrong rea$\operatorname{son}(\mathrm{s})$. Note that choosing menu items is navigation rather than interaction.

For virtually teaching practical skills (and other skills), encourage students to work in pairs (Fenrich, 2005). This is beneficial for extroverts who may otherwise be reluctant to learn via computers. Also, when answering questions, students often discuss the answers, especially when they disagree on which answer is correct. This leads to increased learning. 


\section{Development Phase}

The development phase leads you through creating a storyboard representing the instructional strategy, finalizing the media selection needed to teach the material, and then developing and programming the materials (Fenrich, 2005).

When developing the media, remember that the quality of professionals often shows - it may or may not matter but it usually shows. Follow the maxim that "you get what you pay for".

Using an authoring tool, all of the content must be transferred into a computer program. If the programming shell and templates are designed well, some of this effort can be little more than a cut and paste activity. However, instructional strategies that are relatively unique, such as those needed in virtual labs and simulations, often do not fit within typical templates. However, once something is programmed once, many variations can be relatively easily created.

\section{Evaluation and Revision Phase}

Evaluation is the systematic collection and analysis of information to aid in decision making and planning. A large amount of the evaluation should be done on the paper-based storyboard by content experts and, if possible, target audience learners. It is easier to make changes on paper than on the authoring tool. After this, both the learner's hardware systems (e.g., monitors, memory, computer speed, sound boards ...) and the created software must be evaluated. Keep the following adage in mind, "It is impossible to make things foolproof because fools are so ingenious." Base revisions on the evaluation feedback and data. A major strength of this instructional development cycle model is that evaluation and revision are ongoing throughout the process. This helps prevent changes from becoming too expensive.

\section{Discussion}

By carefully working through the instructional development cycle, following sound principles of instructional design (as should be done when developing all instructional materials), and determining innovative instructional strategies, practical skills can be taught virtually, assuming appropriate resources are available. The key, for teaching practical skills virtually, is creating innovative instructional strategies that address the specified learning outcomes that match, as closely as possible, the skills needed in the real world. When taught well virtually, hands-on skills can transfer to the real world. This does not at all imply that all practical skills can or should be taught virtually. Remember that students do need to practice what is taught in a simulated or actual setting. Imagine trying to learn how to swim without getting your feet wet.

Virtual learning can solve or diminish the inherent distance education problem of teaching handson skills. In some cases, students can learn all they need virtually. In other cases, virtual learning can reduce the amount of time a student needs to spend practicing skills in the traditional lab setting. Alternatively, after virtually learning the skills, the same amount of lab time can be used to bring students to a higher level of ability.

Multimedia technology, in particular the creation of virtual labs, can greatly impact distance education initiatives!

\section{References}

Dick, W., \& Carey, L. (1990). The systematic design of instruction (3rd ed.). Glenville, IL: Harper Collins. Fenrich, P. (2005). Creating instructional multimedia solutions: Practical guidelines for the real world. Self-published. 
Fenrich, P. (2002). An instructional model for teaching troubleshooting skills. Proceedings of the Informing Science and IT Education Conference.

Mayer, M. (1988). Test item construction: A self-instructional manual. Victoria, British Columbia, Canada: Province of British Columbia, Ministry of Advanced Education and Job Training.

McCarthy, B. (1987). The 4MAT system: Teaching to learning styles with right/left mode techniques. Barrington, IL: Excel, Inc.

McFarlane, A., Sparrowhawk, A., \& Heald, Y. (2002). Report on the educational use of games. Teachers Evaluating Educational Multimedia (TEEM), Cambridge, UK. Retrieved November 12, 2004 from http://www.teem.org.uk/publications/teem_gamesined_full.pdf

Romiszowski, A. (1988). The selection and use of instructional media: For improved classroom teaching and for interactive, individual instruction (2nd ed.). New York, NY: Nichols Publishing.

Spitzer, D. (1991). Introduction to instructional technology (2nd ed.). Boise, ID: Boise State University.

\section{Biography}

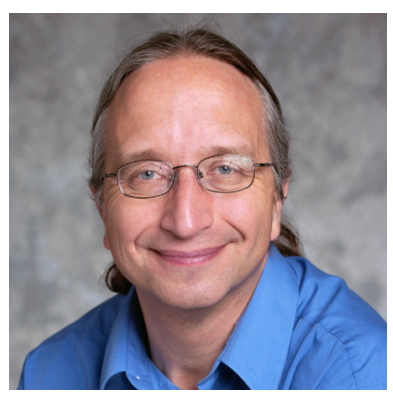

Since 1984, Peter Fenrich has been designing and developing instructional new media products at the British Columbia Institute of Technology (BCIT). His duties include leading projects, writing proposals, designing and developing instructional new media software, conducting research, giving presentations both locally and internationally, training, leading workshops, and evaluating software. His work has been recognized with national and international awards.

Peter teaches a course, both at BCIT and internationally, that he created entitled "Creating Multimedia Instruction". In 1997, Harcourt Brace published his accompanying textbook to the course. He also taught secondary school science and mathematics in Nigeria for two years. 